\title{
ESTABILIDADE DO CREME CONDICIONADOR ALISANTE DE CABELOS, CONTENDO EXTRATO GLICÓLICO DAS CASCAS DE SPONDIAS PURPUREA L. (SERIGÜELA)
}

\author{
Tallys Santana Freitas, Estela Sant'Ana Fachiano, Marcos Alberto Zocoler \\ Universidade do Oeste Paulista - UNOESTE. Curso de Farmácia, Presidente Prudente - SP. zocoler@unoeste.br
}

\section{RESUMO}

O principal ativo utilizado nos alisantes naturais é o tanino, que resulta em um alisamento perfeito. Os taninos são encontrados em diversas plantas, como nas cascas do caule da Spondias purpurea L. (Serigüela). O trabalho tem como objetivo, determinar a estabilidade do creme condicionador alisante contendo extrato glicólico de Spondias purpurea L.. As amostras dos cremes foram armazenadas em temperatura ambiente, geladeira e em estufa. Os testes foram realizados nos tempos 0, 7, 14 e 21 dias. As amostras avaliadas durante o armazenamento, nas condições ambientais e na geladeira, não sofreram modificações em relação à aparência, cor, odor e brilho; porém, as amostras na estufa sofreram intensa modificação da aparência e cor a partir do 14 어 dia. Os valores de $\mathrm{pH}$, não apresentaram alterações acentuadas no decorrer do tempo em análise, demonstrando que o creme manteve-se em pH ácido aceitável para veiculação de extratos contendo taninos.

Palavras-chave: Estabilidade, Spondias purpurea L., Alisantes, Cabelo, Taninos.

\section{STABILITY OF THE STRAIGHTENING HAIR CONDITIONING CREAM, CONTAINING GLYCOLIC EXTRACT FROM THE BARK OF SPONDIAS PURPUREA L. (SERIGUELA)}

\begin{abstract}
The main active straighteners are used in natural tannins, resulting in a perfect smoothing. Tannins are found in various plants as in the stem bark of Spondias purpurea L. (Serigüela). The study aims to determine the stability of smoothing conditioner cream containing glycolic extract of Spondias purpurea $L$.. The samples of the creams were stored at room temperature, refrigerator and oven drying. The tests were performed at 0, 7, 14 and 21 days samples evaluated during storage at ambient conditions and the refrigerator are unchanged with respect to appearance, color, odor and sheen; however the samples in the greenhouse were profoundly modifying the appearance and color from the 14th day. The $\mathrm{pH}$ values showed no marked changes over time in analysis, demonstrando the cream remained at acceptable acid $\mathrm{pH}$ for publication of extracts containing tannins.
\end{abstract}

Keywords: Stability, Spondias purpurea L., straighteners, Hair, Tannins.

\section{INTRODUÇÃO}

Alterar as características naturais do cabelo pode ser esteticamente gratificante, melhora a autoestima, e faz parte de um padrão que a sociedade exige. (LACRIMANTI, 2008).

Segundo DRAELOS (2008), um dos procedimentos frequentemente realizados nos cabelos é o alisamento, o qual pode ser temporário ou definitivo. Os alisamentos temporários, utilizam 
técnicas físico-químicas, como o secador e a piastra ("chapinha"), e também a técnica do "hot comb". Os alisamentos temporários somente duram até a próxima lavagem.

Os alisamentos definitivos visam romper as pontes dissulfeto da queratina. Podem ser à base de hidróxido de sódio, lítio e potássio, hidróxido de guanidina (hidróxido de cálcio mais carbonato de guanidina), bissulfitos e tioglicolato de amônia ou etanolamina, porém são agressivos aos fios de cabelo (SA DIAS et al., 2007).

Os alisantes de cabelos que utilizam ativos naturais não são de uso muito frequente. 0 principal ativo utilizado no alisamento de cabelos é o tanino. Este alisamento resulta num liso perfeito, e tem duração de dois a seis meses, dependendo da manutenção. "Ele serve para todos os tipos de cabelo e não traz dano algum para a saúde". O tempo médio do procedimento é de quatro horas (MODESTO, 2012).

Dentre as diversas plantas contendo taninos em sua composição, destacamos a Spondias purpurea L., conhecida como Serigüela (MARTINS; MELO, 2006).

Zocoler, Vieira e Oliveira (2012) verificaram a partir dos resultados obtidos na análise fitoquímica da droga vegetal de Spondias purpurea L., que esta espécie apresenta taninos e flavonoides nas cascas do caule.

O presente trabalho teve como objetivo o desenvolvimento de creme condicionador alisante de cabelos, contendo extrato glicólico das cascas de Spondias purpurea L. (Serigüela) a $10 \% \mathrm{p} / \mathrm{p}$.

\section{METODOLOGIA}

\section{Coleta, secagem e moagem da Planta}

As cascas do caule de Spondias purpurea L. foram coletadas na cidade de Panorama-SP (latitude 21으, longitude $51^{\circ}$ ) e posteriormente secas em estufa de circulação de ar a uma temperatura de 35 으, por um período de 10 dias. Após a secagem, as cascas foram pulverizadas em moinho de facas. A espécie vegetal foi identificada pelo professor Dr. Décio Gomes de Oliveira, do departamento de Farmacobotânica da Unoeste.

\section{Extração}

O extrato glicólico foi obtido por percolação da droga seca e moída em solução de Propilenoglicol: Água 4:1, durante 3 dias.

\section{Controle de Qualidade do Extrato Glicólico das cascas de Spondias purpurea L.}


Os métodos do controle de qualidade (densidade e $\mathrm{pH}$ ) do extrato glicólico foram realizados segundo a Farmacopéia Brasileira 5ạ ed., 2010.

\section{Creme Condicionar Alisante contendo Extrato Glicólico de Spondias purpurea L.}

A formulação do creme condicionar alisante contendo extrato glicólico de Spondias purpurea L. está descrito abaixo:

Extrato glicólico de Spondias purpurea L...................... 100 $\quad 10$

Cloreto Cetiltrimetil Amônia........................................... $18 \mathrm{~g}$

Álcool Cetoestearílico..................................................

Óleo de Silicone....................................................... $\quad 45 \mathrm{~g}$

Propilparabeno..................................................... $0,9 \mathrm{~g}$

Metilparabeno........................................................ $1,8 \mathrm{~g}$

Propilenoglicol......................................................... 27g

Água purificada qsp................................................. $1000 \mathrm{~g}$

\section{Avaliação da Estabilidade do Creme Condicionador Alisante contendo Extrato Glicólico de Spondias purpurea $L$.}

As amostras de creme condicionador alisante contendo extrato glicólico de Spondias purpurea $L$. foram acondicionadas em potes plásticos, cada uma contendo $100 \mathrm{~g}$, e colocadas nas diferentes condições de armazenamento. As amostras foram armazenadas em três condições distintas: condições ambientais $\left(20^{\circ} \mathrm{C}-25^{\circ} \mathrm{C}\right)$, geladeira (2ㅇ $\left.\mathrm{C}-8^{\circ} \mathrm{C}\right)$ e estufa $\left(45^{\circ} \mathrm{C}\right)$, com temperaturas controladas. Os testes para análise foram realizados nos tempos $0,7,14$ e 21 dias.

As amostras foram analisadas, levando-se em consideração as propriedades organolépticas, através da visualização, considerando qualquer alteração de coloração, odor ou sinal de separação de fases. Para análise dos resultados, serão utilizados critérios apresentados na Tabela 1, adaptado por Bühler e Ferreira (2008).

Tabela 1. Avaliação das características organolépticas

\begin{tabular}{ll}
\hline N & Normal \\
LMA & Leve Modificação da Aparência \\
LMC & Leve Modificação da Cor \\
LMO & Leve Modificação do Odor \\
MA & Modificação da Aparência \\
MC & Modificação da Cor \\
MO & Modificação do Odor \\
IMA & Intensa Modificação da Aparência \\
IMC & Intensa Modificação da Cor \\
IMO & Intensa Modificação do Odor \\
\hline
\end{tabular}

Fonte: Buller e Ferreira, (2008). 
A determinação do $\mathrm{pH}$ foi realizada através do método potenciométrico, utilizando pHmetro digital. $\mathrm{O}$ pH foi determinado nas dispersões das amostras em água recém-destilada na proporção de 1:10 em réplicas de três e à temperatura ambiente $(20,0 \pm 25,0$ 으).

\section{RESULTADOS}

$\mathrm{O} \mathrm{pH}$ do extrato glicólico de Spondias purpurea $L$. foi de 5,4 \pm 0,1; e sua densidade aparente foi de $1,07 \mathrm{~g} / \mathrm{ml} \pm 1,42 \%$

Os resultados da avaliação dos aspectos organolépticos das amostras de creme condicionador alisante contendo extrato glicólico de Spondias purpurea L. analisados durante o período de 21 dias em três condições distintas estão descritos na tabela 2, abaixo.

Tabela 2. Resultados dos aspectos organolépticos das amostras de creme condicionador alisante contendo extrato glicólico de Spondias purpurea $L$. analisados durante o período de 21 dias em três condições distintas

\begin{tabular}{|c|c|c|c|c|c|c|c|c|c|c|c|c|}
\hline \multirow[b]{2}{*}{ Dias } & \multicolumn{4}{|c|}{ Ambiente: $20-25 \circ C$} & \multicolumn{4}{|c|}{ Geladeira: $2-8 \circ \mathrm{C}$} & \multicolumn{4}{|c|}{ Estufa: 45C } \\
\hline & 0 & 7 & 14 & 21 & 0 & 7 & 14 & 21 & 0 & 7 & 14 & 21 \\
\hline Aparência & $\mathrm{N}$ & $\mathrm{N}$ & LMA & LMA & $\mathrm{N}$ & $\mathrm{N}$ & LMA & LMA & $\mathrm{N}$ & $\mathrm{N}$ & IMA & IMA \\
\hline Cor & $\mathrm{N}$ & $\mathrm{N}$ & $\mathrm{N}$ & $\mathrm{N}$ & $\mathrm{N}$ & $\mathrm{N}$ & $\mathrm{N}$ & $\mathrm{N}$ & $\mathrm{N}$ & $\mathrm{N}$ & IMC & IMC \\
\hline Odor & $\mathrm{N}$ & $\mathrm{N}$ & $\mathrm{N}$ & $\mathrm{N}$ & $\mathrm{N}$ & $\mathrm{N}$ & $\mathrm{N}$ & $\mathrm{N}$ & $\mathrm{N}$ & $\mathrm{N}$ & $\mathrm{N}$ & $\mathrm{N}$ \\
\hline
\end{tabular}

Nota: N - normal; LMA - Leve Modificação da Aparência; LMC - leve modificação da cor; LMO - leve modificação do odor; MA - modificação da aparência; MC - modificação da cor; MO - modificação do odor; IMA - intensa modificação da aparência; IMC - intensa modificação da cor

Os valores das medidas do $\mathrm{pH}$ do creme condicionador alisante contendo extrato glicólico de Spondias purpurea L. estão descritos nos gráficos 1, 2 e 3. 
Gráfico 1. Representação gráfica da variação do $\mathrm{pH}$ do creme condicionador alisante contendo extrato glicólico de Spondias purpurea L. analisados durante o período de 21 dias nas condições ambientais.

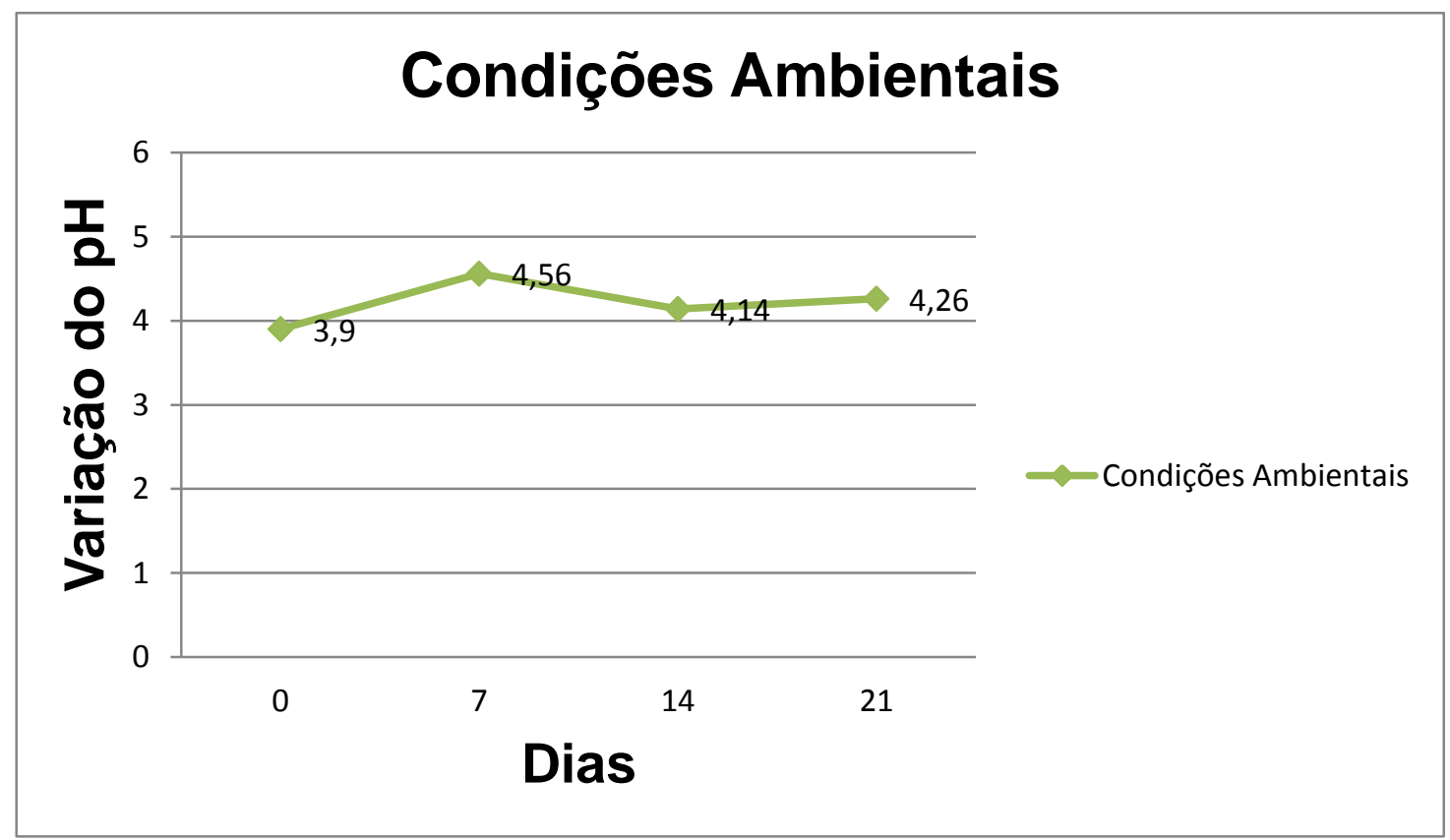

Gráfico 2. Representação gráfica da variação do pH do creme condicionador alisante contendo extrato glicólico de Spondias purpurea $L$. analisados durante o período de 21 dias na geladeira.

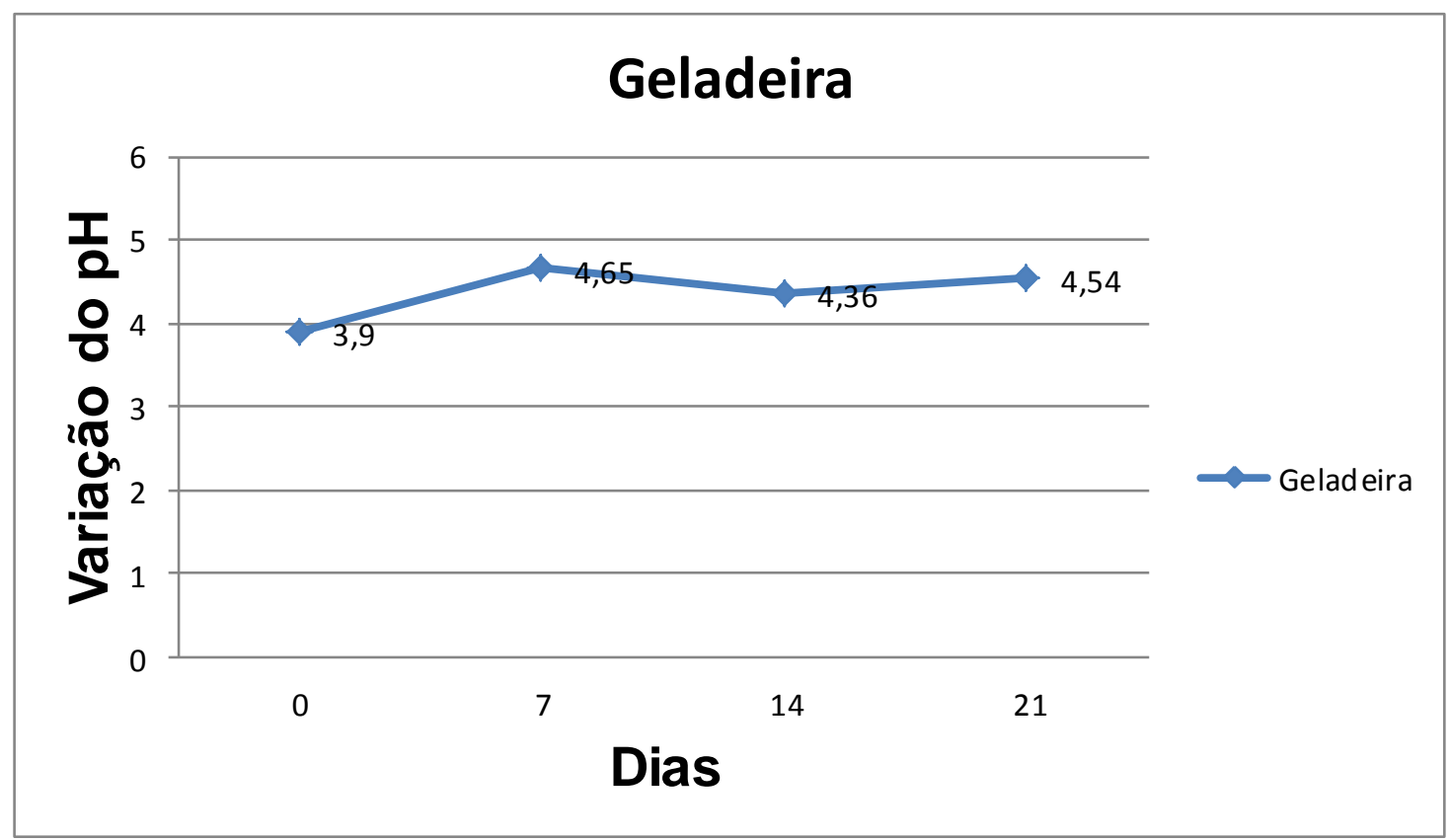


Gráfico 3. Representação gráfica da variação do pH do creme condicionador alisante contendo extrato glicólico de Spondias purpurea L. analisados durante o período de 21 dias na estufa.

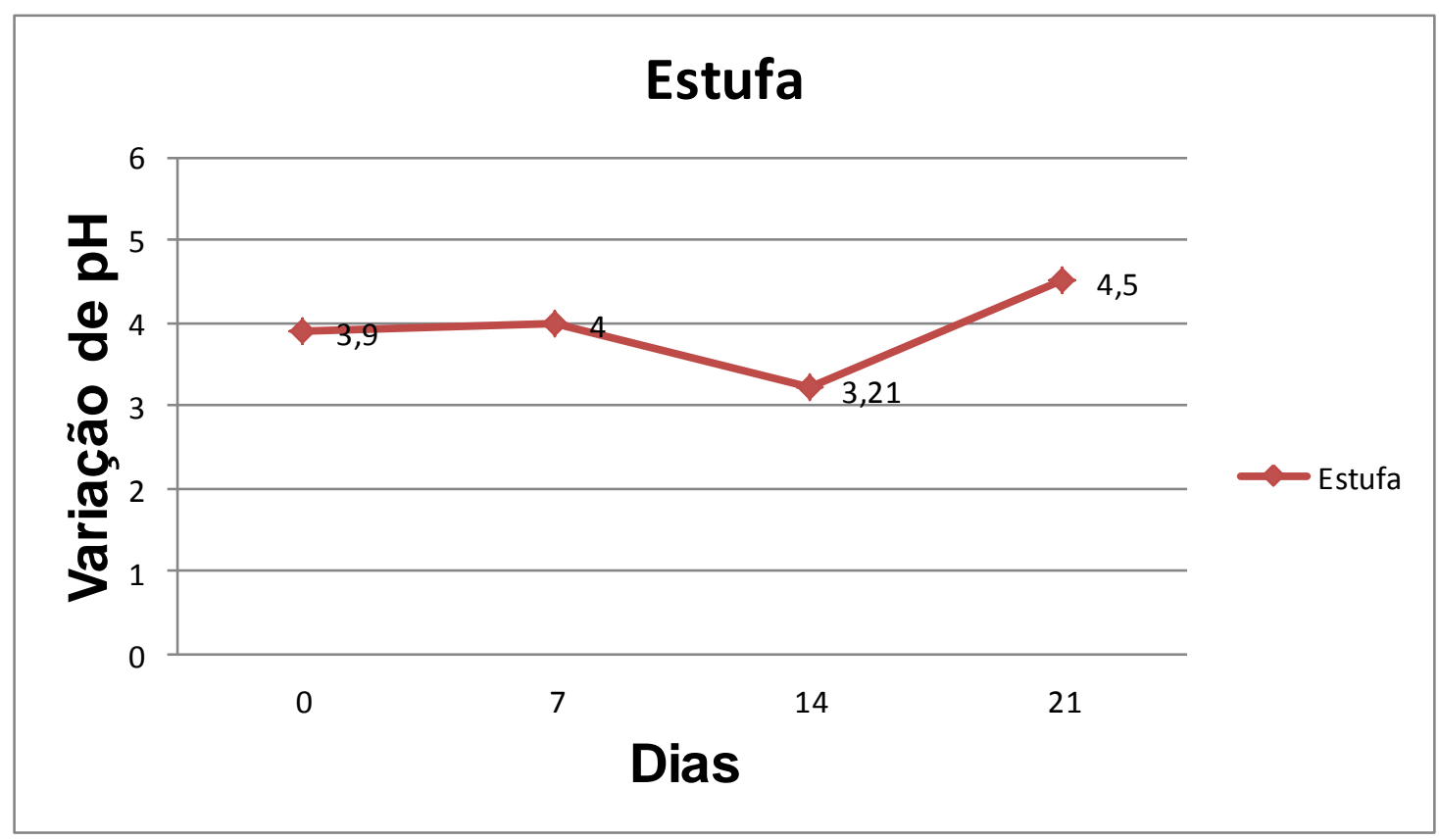

\section{DISCUSSÃO}

A estabilidade de fitocosméticos é definida como o tempo no qual esta se mantém dentro dos limites especificados, ou seja, que durante o período de estocagem e uso, apresentem as mesmas características que possuía quando da época de sua fabricação. A estabilidade dos cosméticos depende de fatores ambientais como temperatura, umidade, luz, $\mathrm{pH}$, condições de armazenagem e de outros fatores relacionados ao próprio produto.

Ensaios organolépticos são procedimentos utilizados para avaliar as características de um produto detectáveis pelos órgãos dos sentidos: aspecto, cor, odor e tato. Eles fornecem parâmetros que permitem avaliar, de imediato, o estado da amostra em estudo por meio de análises comparativas, com o objetivo de verificar alterações como separação de fases, precipitação e turvação, possibilitando o reconhecimento primário do produto (BRASIL, 2004).

As amostras do creme condicionador alisante contendo extrato glicólico de Spondias purpurea $L$. nas condições ambientais e na geladeira não sofreram modificações significativas em relação à aparência, cor, odor e brilho. Com relação às amostras que permaneceram na estufa, apresentaram a partir da segunda semana, intensa modificação na aparência e na coloração, indicando uma possível oxidação dos componentes do extrato glicólico de Spondias purpurea L.. Em relação ao odor, este permaneceu constante durante o ensaio na estufa.

Das amostras de creme condicionador alisante contendo extrato glicólico de Spondias purpurea, L. os resultados da Tabela 2 indicam que em temperaturas mais elevadas (estufa $-45^{\circ} \mathrm{C}$ ) 
os componentes da formulação têm probabilidade de se tornarem instáveis. Embora as modificações sugeridas a partir dessas amostras sejam aceitáveis devido à condição em que foram armazenadas, as características organolépticas do produto em questão foram relativamente modificadas. Dessa forma, as amostras mantidas em estufa vão contra a aceitação do consumidor caso o creme viesse a ser comercializado.

O estudo da estabilidade contribui para orientar o desenvolvimento da formulação e do material de acondicionamento; fornecer subsídios para aperfeiçoamento das formulações; estimar o prazo de validade e fornecer informações para sua confirmação; auxiliar no monitoramento da estabilidade organoléptica, físico-química e microbiológica, produzindo informações sobre a confiabilidade e segurança dos produtos. O teste de estabilidade preliminar, realizado em um curto intervalo de tempo, pode ser considerado um teste orientativo no desenvolvimento de produtos. (ISAAC et al., 2008).

Um dos fatores que afeta a estabilidade dos cremes é o $\mathrm{pH}$, pois ele determina pontos onde ocorre ou não a formação precoce de processos de oxidação e hidrólise do material oleoso, formando o ranço e outros materiais (ATKINS, 1999). Portanto a avaliação do pH é um parâmetro essencial na estabilidade das emulsões.

Os valores de $\mathrm{pH}$, não apresentaram alterações acentuadas no decorrer do tempo em análise, sugerindo comportamento relativamente estável de pH frente às condições testadas em ambas as análises. As amostras permaneceram em pH ácido, com leves alterações no decorrer do tempo. Indicando que a base é ideal para a estabilidade de ativos ácidos, como os compostos fenólicos presentes no extrato glicólico de Spondias purpurea $L$.

$\mathrm{O}$ pH também é essencial para manter a estabilidade dos metabólitos secundários presentes nos extratos vegetais, utilizados como matéria-prima na obtenção de pomadas fitoterápicas. A degradação de muitos metabólitos secundários é catalisada pelo pH extremo, ou seja, muito ácido ( $\mathrm{pH}$ menor que 2,0) ou muito básico ( $\mathrm{pH}$ maior que 8,0). A maioria dos metabólitos secundários é estável na faixa de pH entre 4,0 a 6,0 (AULTON, 2006).

\section{CONCLUSÃO}

A partir dos resultados obtidos, verificamos que a base do creme condicionador apresenta boa estabilidade para incorporação de fármacos, extratos e tinturas. O creme condicionador alisante contendo extrato glicólico de Spondias purpurea $L$. apresenta boa estabilidade em relação ao $\mathrm{pH}$ e às características organolépticas quando armazenado em geladeira e na temperatura 
ambiente. Já quando armazenado em temperaturas elevadas, suas características organolépticas, nessas condições, apresentaram alterações significativas que impedem sua comercialização.

\section{REFERÊNCIAS}

1. ATKINS, F.W. Físico-Química. Rio de Janeiro: Livros Técnicos, p. 336-342. 1999.

2. AULTON, M. E. Delineamento de formas farmacêuticas. 2. ed. Porto Alegre: Artmed, 2006.

3. BRASIL. Ministério da Saúde. Guia de Estabilidade de Produtos Cosméticos. Agência Nacional de Vigilância Sanitária, Brasília, 2004. Disponível em: <http://www.anvisa.gov.br/divulga/public/series/cosmeticos.pdf>. Acesso em: 13 jun. 2014.

4. DRAELOS ZD. "Hair Cosmetics". In: Blume-Peytav U, Tosti A et al. Hair Growth and Disorders. Springer. 1 ed. 2008.

5. FARMACOPÉIA BRASILEIRA. 5a ed., Brasília, DF: Anvisa: Fundação Oswaldo Cruz, 2010. Disponível em: <www.anvisa.gov.br/hotsite/cd_farmacopeia/index.htm/>. Acesso em: 12 mar. 2011.

6. ISAAC, V. L. B. et al. Protocolo para ensaios físico-químicos de estabilidade de fitocosméticos. Rev. Ciênc. Farm. Básica Apl., Araraquara, v. 29, n. 1, jul., 2008. Disponível em: <http://serv-bib.fcfar.unesp.br/seer/index.php/Cien_Farm/article/view/438/421>. Acesso em: 25 jun. 2014.

7. LACRIMANTI, Lígia Marini. Curso didático de estética: v. 2. São Caetano do Sul: Yendis, 2008.

8. MARTINS, S. T.; MELO, B. Spondias (Cajá e outras). 2006. Disponível em: <http://www.fruticultura.iciag.ufu.br/caja.html>. Acesso em 13 mar. 2011.

9. MODESTO C. Alisamento natural é a nova onda. Correio da Paraíba - PB 16/09/2012. Disponivel em: <file:///C:/Users/usuarios/Downloads/noticia 18893794.pdf > Acesso em: 15 maio 2014.

10. SÁ DIAS TC, BABY AR, KANKO TM et al. Relaxing/straightenig of Afro-ethinic hair: historical overview. J Cosmet Dermatol 2007.

11. ZOCOLER, M.A.; VIEIRA, J.T.M.; OLIVEIRA, J.M. Avaliação farmacognóstica e triagem fitoquímica da droga vegetal e do extrato bruto de folhas e cascas de Spondias purpurea (Siriguela). 2012. Trabalho de Conclusão de Curso. Unoeste. 